\title{
REGULARIZATION OF CURRENTS AND ENTROPY
}

\author{
By TIEN-CUONG DINH AND NESSIM SIBONY
}

\begin{abstract}
Let $T$ be a positive closed $(p, p)$-current on a compact Kähler manifold $X$. We prove the existence of smooth positive closed $(p, p)$-forms $T_{n}^{+}$and $T_{n}^{-}$such that $T_{n}^{+}-T_{n}^{-} \rightarrow T$ weakly. Moreover, $\left\|T_{n}^{ \pm}\right\| \leqslant c_{X}\|T\|$ where $c_{X}>0$ is a constant independent of $T$. We also extend this result to positive pluriharmonic currents. Then we study the wedge product of positive closed $(1,1)$-currents having continuous potential with positive pluriharmonic currents. As an application, we give an estimate for the topological entropy of meromorphic maps on compact Kähler manifolds.
\end{abstract}

() 2004 Elsevier SAS

RÉSUMÉ. - Soit $T$ un $(p, p)$-courant positif fermé sur une variété kählérienne compacte $X$. Nous montrons l'existence de $(p, p)$-formes lisses, positives fermées $T_{n}^{+}$et $T_{n}^{-}$telles que $T_{n}^{+}-T_{n}^{-} \rightarrow T$ faiblement. De plus, on a $\left\|T_{n}^{ \pm}\right\| \leqslant c_{X}\|T\|$ où $c_{X}>0$ est une constante indépendante de $T$. Nous montrons aussi ce résultat pour les courants positifs pluriharmoniques. Nous étudions également le produit extérieur de $(1,1)$-courants positifs fermés à potentiel continu avec des courants pluriharmoniques positifs. Comme application, nous donnons une estimation de l'entropie topologique des applications méromorphes d'une variété kählérienne compacte.

(ㄷ) 2004 Elsevier SAS

\section{Introduction}

Let $(X, \omega)$ be a compact Kähler manifold of dimension $k$. Demailly [7] has shown that for a positive closed $(1,1)$-current $T$ on $X$, there exist smooth positive closed $(1,1)$-forms $T_{n}^{+}$which converge weakly (i.e. in the sense of currents) to $T+c \omega$ where $c>0$ is a constant. Moreover, there is a constant $c_{X}>0$, independent of $T$, such that $\left\|T_{n}^{+}\right\|$and $c$ are bounded by $c_{X}\|T\|$. We refer to Demailly's papers [6,7] for the basics on currents on complex manifolds. Recall that the mass of a positive $(p, p)$-current $S$ is defined by $\|S\|:=\int_{X} S \wedge \omega^{k-p}$. Our main result is the following theorem where positivity can be understood in the weak or strong sense.

THEOREM 1.1. - Let $(X, \omega)$ be a compact Kähler manifold of dimension $k$. Then, for every positive closed $(p, p)$-current $T$ on $X$, there exist smooth positive closed $(p, p)$-forms $T_{n}^{+}$and $T_{n}^{-}$such that $T_{n}^{+}-T_{n}^{-}$converge weakly to the current $T$. Moreover, $\left\|T_{n}^{ \pm}\right\| \leqslant c_{X}\|T\|$ where $c_{X}>0$ is a constant independent of $T$.

We deduce from this theorem the following corollary which is proved in [10] for projective manifolds.

COROLlary 1.2. - Let $(X, \omega)$ be a compact Kähler manifold of dimension $k$. Then, for every positive closed $(p, p)$-current $T$ on $X$, there exist smooth positive closed $(p, p)$-forms $T_{n}^{+}$which converge weakly to a current $T^{\prime}$ with $T^{\prime} \geqslant T$. Moreover, $\left\|T_{n}^{+}\right\| \leqslant c_{X}\|T\|$ and $\left\|T^{\prime}\right\| \leqslant c_{X}\|T\|$ where $c_{X}>0$ is a constant independent of $T$. 
Let $\left(X^{\prime}, \omega^{\prime}\right)$ be another compact Kähler manifold of dimension $k^{\prime} \geqslant k$ and let $\Pi: X^{\prime} \rightarrow X$ be a surjective holomorphic map. We want to define the pull-back of the current $T$ by the map $\Pi$. When $\Pi$ is a finite map, this problem is studied in [19,12]. In general, the map $\Pi$ is a submersion only in the complement of an analytic subset $\mathrm{C}$ of $X^{\prime}$. Let $\pi$ denote the restriction of $\Pi$ to $X^{\prime} \backslash \mathrm{C}$. Then, $\pi^{*}(T)$ is well defined and is a positive closed $(p, p)$-current on $X^{\prime} \backslash$ C. Let $\left(T_{n}^{+}\right)$and $c_{X}$ be as in Corollary 1.2. Define $S_{n}:=\Pi^{*}\left(T_{n}^{+}\right)$. The $(p, p)$-forms $S_{n}$ are smooth and positive on $X^{\prime}$. Their classes in $H^{p, p}\left(X^{\prime}, \mathbb{C}\right)$ are bounded since $\left(\left\|T_{n}^{+}\right\|\right)$is bounded. It follows that $\left(\left\|S_{n}\right\|\right)$ is bounded. Taking a subsequence, we can assume that $S_{n}$ converge to a current $S$. We also have $S \geqslant \pi^{*}(T)$ on $X^{\prime} \backslash$ C. In particular, $\pi^{*}(T)$ has finite mass. Following Skoda [22], the trivial extension $\widetilde{\pi^{*}(T)}$ of $\pi^{*}(T)$ on $X^{\prime}$ is a positive closed current. So, we have the following corollary.

COROLlary 1.3. - Let $X, X^{\prime}, \Pi, \pi$ and $T$ be as above. Then, the positive current $\widetilde{\pi^{*}(T)}$ is well defined and closed. Moreover, there exists a constant $c_{\Pi}>0$ independent of $T$ such that $\left\|\pi^{*}(T)\right\| \leqslant c_{\Pi}\|T\|$. The map $T \mapsto \widehat{\pi^{*}(T)}$ is l.s.c. in the sense that if $T_{n} \rightarrow T$, then any cluster point $\tau$ of $\left(\widetilde{\pi^{*}\left(T_{n}\right)}\right)$ satisfies $\tau \geqslant \widehat{\pi^{*}(T)}$.

In [19], Méo gave an example which shows that, in general, when $X$ and $X^{\prime}$ are not compact, the current $\pi^{*}(T)$ on $X \backslash \mathrm{C}$ is not always of bounded mass near C.

Consider a dominating meromorphic self-map $f: X \rightarrow X$ of $X$. Define $f^{n}:=f \circ \cdots \circ f$ ( $n$ times) the $n$th iterate of $f$. We refer to the survey [20] for the theory of iteration of meromorphic maps. Let $I_{n}$ be the indeterminacy set of $f^{n}$. Then $I_{n}$ is an analytic subset of codimension $\geqslant 2$ of $X$. Denote by $\Omega_{f}$ the set of points $x \in X \backslash I_{1}$ such that $f^{n}(x) \notin I_{1}$ for every $n \geqslant 1$. A subset $F \subset \Omega_{f}$ is called $(n, \epsilon)$-separated, $\epsilon>0$, if

$$
\max _{0 \leqslant i \leqslant n-1} \operatorname{dist}\left(f^{i}(x), f^{i}(y)\right) \geqslant \epsilon \quad \text { for } x, y \in F \text { distinct. }
$$

The topological entropy $\mathrm{h}(f)$ (see [5]) is defined by

$$
\mathrm{h}(f):=\sup _{\epsilon>0}\left(\limsup _{n \rightarrow \infty} \frac{1}{n} \log \max \{\# F, F(n, \epsilon) \text {-separated }\}\right) .
$$

Let $\Gamma_{n}$ be the closure in $X^{n}$ of the set of points

$$
\left(x, f(x), \ldots, f^{n-1}(x)\right), \quad x \in \Omega_{f} .
$$

This is an analytic subset of dimension $k$ of $X^{n}$. Let $\Pi_{i}$ be the canonical projections of $X^{n}$ on its factors. We consider on $X^{n}$ the Kähler metric $\omega_{n}:=\sum \Pi_{i}^{*}(\omega)$. Define, following Gromov [17],

$$
\operatorname{lov}(f):=\limsup _{n \rightarrow \infty} \frac{1}{n} \log \left(\operatorname{vol}\left(\Gamma_{n}\right)\right)=\limsup _{n \rightarrow \infty} \frac{1}{n} \log \left(\int_{\Gamma_{n}} \omega_{n}^{k}\right) .
$$

Define also the dynamical degree of order $p$ of $f$ by

$$
d_{p}:=\limsup _{n \rightarrow \infty}\left(\int_{X \backslash I_{n}} f^{n *}\left(\omega^{p}\right) \wedge \omega^{k-p}\right)^{1 / n} .
$$


Using an inequality of Lelong [18], Gromov [17] proved that $\mathrm{h}(f) \leqslant \operatorname{lov}(f)$. Following Gromov and Yomdin [23,16,17], we have

$$
\mathrm{h}(f)=\operatorname{lov}(f)=\max _{1 \leqslant p \leqslant k} \log d_{p}
$$

when $f$ is a holomorphic map. Using Corollary 1.2, we prove, in the same way as in [10], that the sequences in (1), (2) are convergent and that the dynamical degrees $d_{p}$ are bimeromorphic invariants of $f$. More precisely, if $\Pi: X^{\prime} \rightarrow X$ is a bimeromorphic map between compact Kähler manifolds, the dynamical degrees of $\Pi^{-1} \circ f \circ \Pi$ are equal to $d_{p}$. Using Corollary 1.2, we also get the following result.

THEOREM 1.4. - Let $f$ be a dominating meromorphic self-map on a compact Kähler manifold $X$ of dimension $k$. Let $d_{p}$ be the dynamical degrees of $f$. Then

$$
\mathrm{h}(f) \leqslant \operatorname{lov}(f)=\max _{1 \leqslant p \leqslant k} \log d_{p}
$$

This theorem gives a partial answer to a conjecture of Friedland [15] which says that $\mathrm{h}(f)=\max _{1 \leqslant p \leqslant k} \log d_{p}$. Theorem 1.4 is already proved in [10] for rational maps on projective manifolds. Corollary 1.2 permits to extend the proof to compact Kähler manifolds. One can also extend some results on meromorphic correspondences or transforms, which are proved in the projective case in [11] (see also [8]).

In the last two sections, we extend Theorem 1.1 to positive pluriharmonic currents and currents of class DSH. We also study the intersection of such currents with positive closed $(1,1)$-currents.

We thank the referee for his constructive observations that helped to improve the exposition.

\section{A classical lemma}

We give here a classical lemma that we use in Section 3. Let $B$ denote the unit ball in $\mathbb{R}^{m}$. Let $K(x, y)$ be a function with compact support in $\mathrm{B} \times \mathrm{B}$, smooth in $\mathrm{B} \times \mathrm{B} \backslash \Delta$ where $\Delta$ is the diagonal of $\mathrm{B} \times \mathrm{B}$. Assume that, for every $(x, y)$

$$
|K(x, y)| \leqslant A|x-y|^{2-m}
$$

where $A>0$ is a constant and $x=\left(x_{1}, \ldots, x_{m}\right)$ are coordinates of $\mathbb{R}^{m}$. Observe that for every $y$

$$
\|K(., y)\|_{\mathrm{L}^{1+\delta}} \leqslant A^{\prime}
$$

for some $\delta>0$ and $A^{\prime}>0$. Assume also that for every $x, y$

$$
|\nabla K(x, y)| \leqslant A|x-y|^{1-m}
$$

In this section, we identify $\nu$, a current of degree 0 and of order 0 , with the current of degree $m$, $\nu \mathrm{d} y_{1} \wedge \cdots \wedge \mathrm{d} y_{m}$. Let $\mathcal{M}$ denote the set of Radon measures on $\mathbb{R}^{m}$. We define a linear operator $P$ on $\mathcal{M}$ by:

$$
P \mu(x):=\int_{y \in \mathbb{R}^{m}} K(x, y) \mathrm{d} \mu(y) .
$$

Observe that the function $P \mu$ has support in B. We have the following lemma. 
LEMMA 2.1. - The operator $P$ maps continuously $\mathcal{M}$ into $\mathrm{L}^{1+\delta}$. It also maps continuously $\mathrm{L}^{p}$ into $\mathrm{L}^{q}, \mathrm{~L}^{\infty}$ into $\mathcal{C}^{0}$ and $\mathcal{C}^{0}$ into $\mathcal{C}^{1}$, where $q=\infty$ if $p^{-1}+(1+\delta)^{-1} \leqslant 1$ and $p^{-1}+(1+\delta)^{-1}=$ $1+q^{-1}$ otherwise.

All the assertions are easy to deduce from (3), (4) and (5) and the Hölder inequality.

\section{Proof of Theorem 1.1}

Let $\Delta$ denote the diagonal of $X \times X$. We first give a weak regularization of the current of integration $[\Delta]$. Let $\overline{X \times X}$ denote the blow-up of $X \times X$ along $\Delta$. Following Blanchard [4], $\widehat{X \times X}$ is a Kähler manifold. Let $\pi: \widehat{X \times X} \rightarrow X \times X$ be the canonical projection and $\widetilde{\Delta}:=\pi^{-1}(\Delta)$. Then $\widetilde{\Delta}$ is a smooth hypersurface in $\widehat{X \times X}$. If $\gamma$ is a closed strictly positive $(k-1, k-1)$-form on $\widetilde{X \times X}$, then $\pi_{*}(\gamma \wedge[\widetilde{\Delta}])$ is a non-zero positive closed $(k, k)$-current on $X \times X$ supported on $\Delta$. So, it is a multiple of $[\Delta]$. We choose $\gamma$ so that $\pi_{*}(\gamma \wedge[\widetilde{\Delta}])=[\Delta]$. We will use the following regularization of $[\widetilde{\Delta}]$.

Since $[\widetilde{\Delta}]$ is a positive closed $(1,1)$-current, there exist a quasi-p.s.h. function $\varphi$ and a smooth closed $(1,1)$-form $\Theta^{\prime}$ such that $\mathrm{dd}^{\mathrm{c}} \varphi=[\widetilde{\Delta}]-\Theta^{\prime}$. Recall that $\mathrm{d}^{\mathrm{c}}:=\frac{i}{2 \pi}(\bar{\partial}-\partial)$. Demailly's regularization theorem [7] implies the existence of smooth functions $\varphi_{n}$ and of a smooth positive closed $(1,1)$-form $\Theta$ on $\widetilde{X \times X}$ such that

- $\operatorname{dd}^{\mathrm{c}} \varphi_{n} \geqslant-\Theta$

- $\varphi_{n}$ decrease to $\varphi$.

In this case, independently of Demailly's theorem, we can construct the functions $\varphi_{n}$ as follows. Observe that $\varphi$ is smooth out of $\widetilde{\Delta}$ and $\varphi^{-1}(-\infty)=\widetilde{\Delta}$. Let $\chi: \mathbb{R} \cup\{-\infty\} \rightarrow \mathbb{R}$ be a smooth increasing convex function such that $\chi(x)=0$ on $[-\infty,-1], \chi(x)=x$ on $[1,+\infty[$ and $0 \leqslant \chi^{\prime} \leqslant 1$. Define $\chi_{n}(x):=\chi(x+n)-n$ and $\varphi_{n}:=\chi_{n} \circ \varphi$. The functions $\varphi_{n}$ are smooth decreasing to $\varphi$ and we have

$$
\begin{aligned}
\operatorname{dd}^{\mathrm{c}} \varphi_{n} & =\left(\chi_{n}^{\prime \prime} \circ \varphi\right) \mathrm{d} \varphi \wedge \mathrm{d}^{\mathrm{c}} \varphi+\left(\chi_{n}^{\prime} \circ \varphi\right) \operatorname{dd}^{\mathrm{c}} \varphi \\
& \geqslant\left(\chi_{n}^{\prime} \circ \varphi\right) \mathrm{dd}^{\mathrm{c}} \varphi=-\left(\chi_{n}^{\prime} \circ \varphi\right) \Theta^{\prime} \geqslant-\Theta
\end{aligned}
$$

where we choose the smooth positive closed form $\Theta$ big enough so that $\Theta-\Theta^{\prime}$ is positive.

Define $\Theta_{n}^{+}:=\operatorname{dd}^{\mathrm{c}} \varphi_{n}+\Theta$ and $\Theta_{n}^{-}:=\Theta-\Theta^{\prime}$ then $\Theta_{n}^{+}-\Theta_{n}^{-} \rightarrow[\widetilde{\Delta}]$. We have $\left\|\Theta_{n}^{ \pm}\right\| \leqslant c_{0}$ where $c_{0}>0$ is a constant. The forms $\Theta_{n}^{ \pm}$are smooth. Define

$$
\widetilde{K}_{n}^{ \pm}:=\gamma \wedge \Theta_{n}^{ \pm} \quad \text { and } \quad K_{n}^{ \pm}:=\pi_{*}\left(\widetilde{K}_{n}^{ \pm}\right)
$$

The $(k, k)$-forms $K_{n}^{ \pm}$are positive closed with coefficients in $\mathrm{L}^{1}$ and smooth out of $\Delta$. We also have $K_{n}^{+}-K_{n}^{-} \rightarrow[\Delta]$ weakly and $\left\|K_{n}^{ \pm}\right\| \leqslant c_{1}, c_{1}>0$. This is what we call a weak regularization of $[\Delta]$. We will use $K_{n}^{ \pm}$to regularize the current $T$. The following lemma shows that the coefficients of $K_{n}^{ \pm}$satisfy inequalities of type (3) and (5) for $m=2 k$. Then, the singularities of $K_{n}^{ \pm}$are the same as the singularities of the Bochner-Martinelli kernel.

LEMMA 3.1. - Let $(x, y)=\left(x_{1}, \ldots, x_{k}, y_{1}, \ldots, y_{k}\right),\left|x_{i}\right|<3,\left|y_{i}\right|<3$, be local holomorphic coordinates of a chart of $X \times X$ such that $\Delta=(y=0)$ in that chart. Let $H_{n}^{ \pm}$be a coefficient of $K_{n}^{ \pm}$in these coordinates. Then, there exists a constant $A_{n}>0$, depending on $n$, such that

$$
\left|H_{n}^{ \pm}(x, y)\right| \leqslant A_{n}|y|^{2-2 k} \quad \text { and } \quad\left|\nabla H_{n}^{ \pm}\right| \leqslant A_{n}|y|^{1-2 k}
$$

for $\left|x_{i}\right| \leqslant 1,\left|y_{i}\right| \leqslant 1$ and $y \neq 0$. 
Proof. - By symmetry, it is sufficient to consider $(x, y)$ in the open sector $S$ defined by the inequalities $\left|x_{i}\right|<3,\left|y_{i}\right|<3,\left|y_{i}\right|<3\left|y_{1}\right|$ and prove the estimates in the sector $S^{\prime}$ defined by $\left|x_{i}\right|<2,\left|y_{i}\right|<2$ and $\left|y_{i}\right|<2\left|y_{1}\right|$ (we can assume that $y_{1}$ is the largest coordinate of the point $y \neq 0)$. Let $\widetilde{S}$ and $\widetilde{S}^{\prime}$ be the interiors of $\pi^{-1}(\bar{S})$ and of $\pi^{-1}\left(\bar{S}^{\prime}\right)$ respectively. We consider the coordinate system $(x, Y)$ of $\widetilde{S}$ with $\left|x_{i}\right|<3, Y_{1}=y_{1}$ and $Y_{i}=y_{i} / y_{1},\left|y_{1}\right|<3,\left|y_{i}\right|<3\left|y_{1}\right|$ for $i=2, \ldots, k$. We have $\pi(x, Y)=(x, y)$ for $(x, y) \in S$. The equation of $\widetilde{\Delta}$ in $\widetilde{S}$ is $Y_{1}=0$.

Since $\widetilde{K}_{n}^{ \pm}$are smooth on $\widetilde{S}$, they are finite sums of forms of type

$$
\Phi(x, Y)=L(x, Y) \mathrm{d} x_{I} \wedge \mathrm{d} \bar{x}_{I^{\prime}} \wedge \mathrm{d} Y_{J} \wedge \mathrm{d} \bar{Y}_{J^{\prime}}
$$

where $L$ is a smooth function, $I, I^{\prime}, J, J^{\prime}$ are subsequences of $\{1, \ldots, k\}$ and

$$
\mathrm{d} x_{I}=\mathrm{d} x_{i_{1}} \wedge \cdots \wedge \mathrm{d} x_{i_{m}} \text { if } I=\left\{i_{1}, \ldots, i_{m}\right\} .
$$

Hence, in $S$ the forms $K_{n}^{ \pm}$are finite sums of forms of type $\pi_{*}(\Phi)$.

Observe that $\pi_{*}(\Phi)$ is obtained from $\Phi(x, Y)$ replacing $Y_{1}$ by $y_{1}$ and $Y_{i}$ by $y_{i} / y_{1}$. There are here at most $2 k-2$ factors of the form $\mathrm{d}\left(y_{i} / y_{1}\right)=\mathrm{d} y_{i} / y_{1}-y_{i} \mathrm{~d} y_{1} / y_{1}^{2}$ or their conjugate. Hence, the coefficients of $\pi_{*}(\Phi)$ on $S$ are finite sums of

$$
L\left(x, y_{1}, y_{2} / y_{1}, \ldots, y_{k} / y_{1}\right) P(y) y_{1}^{-m} \bar{y}_{1}^{-n}
$$

where $P$ is a homogeneous polynomial in $y$ and $\bar{y}$ such that $\operatorname{deg}(P)+2 k-2 \geqslant m+n$. Since $\widetilde{S^{\prime}} \Subset \widetilde{S}, L$ is bounded on $\widetilde{S}^{\prime}$ and $L\left(x, y_{1}, y_{2} / y_{1}, \ldots, y_{k} / y_{1}\right)$ is bounded on $S^{\prime}$. The first estimate of the lemma follows.

For the second estimate, it is sufficient to observe that the coefficients in the gradient of

$$
L\left(x, y_{1}, y_{2} / y_{1}, \ldots, y_{k} / y_{1}\right) P(y) y_{1}^{-m} \bar{y}_{1}^{-n}
$$

are combinations of functions of the same type with homogeneous polynomials $P$ such that $\operatorname{deg}(P)+2 k-1 \geqslant m+n$.

Define

$$
T_{n}^{ \pm}(x):=\int_{y \in X} K_{n}^{ \pm}(x, y) \wedge T(y) .
$$

Let $\pi_{i}$ denote the canonical projections of $X \times X$ on its factors. We have

$$
T_{n}^{ \pm}:=\left(\pi_{1}\right)_{*}\left(K_{n}^{ \pm} \wedge \pi_{2}^{*}(T)\right) .
$$

Observe that $\pi_{2}^{*}(T)$ is well defined since $\pi_{2}$ is a submersion. The currents $K_{n}^{ \pm} \wedge \pi_{2}^{*}(T)$ are positive closed and well defined on $X \times X \backslash \Delta$. They are of finite mass since, for each $n$, $\left\|K_{n}^{+}(., y)\right\|_{\mathrm{L}^{1}}$ is uniformly bounded. A priori, the mass depends on $n$. By Skoda's extension theorem [22], their trivial extensions are positive and closed. It follows that $T_{n}^{ \pm}$are well defined and are positive closed currents on $X$. The use of Skoda's theorem can be replaced by an argument similar to the one in the proof of the following lemma.

Lemma 3.2. - The currents $T_{n}^{+}-T_{n}^{-}$converge weakly to $T$ when $n \rightarrow \infty$. Moreover, $\left\|T_{n}^{ \pm}\right\| \leqslant c\|T\|$ where $c>0$ is a constant independent of $n$ and $T$. 
Proof. - Define $\Pi:=\pi_{2} \circ \pi$. Observe that $\Pi$ is a submersion from $\widetilde{X \times X}$ onto $X$ and $\Pi_{\mid \widetilde{\Delta}}$ is a submersion from $\widetilde{\Delta}$ onto $X$. Indeed, consider charts $U \Subset V^{\prime} \subset X$ that we identify with open sets in $\mathbb{C}^{k}$. Assume that $U$ is small enough and $0 \in U$. We can, using the change of coordinates $(z, w) \mapsto(z-w, w)$ on $V^{\prime} \times U$, reduce to the product situation $V \times U, U \Subset V \subset \mathbb{C}^{k}$ where $\Delta$ is identified to $\{0\} \times U$. The blow-up along $\{0\} \times U$ is still a product. So $\Pi^{*}$ of a current is just integration on fibers. We can use this local model for the assertions below.

The potential of $\widetilde{\Delta}$ is integrable with respect to $\Pi^{*}(T)$ since its singularity is like log $\operatorname{dist}(z, \widetilde{\Delta})$ and this function has bounded integral on fibers of $\Pi$. In particular, $[\widetilde{\Delta}] \wedge \Pi^{*}(T)$ is well defined (we use here that $T$ is closed) and is equal to $\left(\Pi_{\mid \widetilde{\Delta}}\right)^{*}(T)$, and $[\widetilde{\Delta}]$ has no mass for $\Pi^{*}(T)$ nor for $\widetilde{K}_{n}^{ \pm} \wedge \Pi^{*}(T)$. We then have

$$
K_{n}^{ \pm} \wedge \pi_{2}^{*}(T)=\pi_{*}\left(\widetilde{K}_{n}^{ \pm} \wedge \Pi^{*}(T)\right)
$$

since the formula is valid out of $\Delta$ and there is no mass on $\Delta$. The potentials of $\widetilde{K}_{n}^{+}$are decreasing and the currents $\widetilde{K}_{n}^{-}$are independent of $n$, hence

$$
\widetilde{K}_{n}^{+} \wedge \Pi^{*}(T)-\widetilde{K}_{n}^{-} \wedge \Pi^{*}(T) \rightarrow \gamma \wedge[\widetilde{\Delta}] \wedge \Pi^{*}(T)=\gamma \wedge\left(\Pi_{\mid \widetilde{\Delta}}\right)^{*}(T)
$$

Since $\pi_{\mid \widetilde{\Delta}}$ is a submersion onto $\Delta$, we have $\left(\Pi_{\mid \widetilde{\Delta}}\right)^{*}(T)=\left(\pi_{\mid \widetilde{\Delta}}\right)^{*}\left(\pi_{2 \mid \Delta}\right)^{*}(T)$. Hence

$$
\pi_{*}\left(\gamma \wedge\left(\Pi_{\mid \widetilde{\Delta}}\right)^{*}(T)\right)=\left(\pi_{2 \mid \Delta}\right)^{*}(T)
$$

This and (9), (10) imply that

$$
K_{n}^{+} \wedge \pi_{2}^{*}(T)-K_{n}^{-} \wedge \pi_{2}^{*}(T) \rightarrow\left(\pi_{2 \mid \Delta}\right)^{*}(T) .
$$

Taking the direct image under $\pi_{1}$ gives $T_{n}^{+}-T_{n}^{-} \rightarrow T$.

Since $\Pi$ is a submersion, $\left\|\Pi^{*}(T)\right\| \leqslant c_{2}\|T\|$ where $c_{2}>0$ is independent of $T$. Observe that since $\widetilde{K}_{n}^{ \pm}$are smooth we can compute $\left\|\widetilde{K}_{n}^{ \pm} \wedge \Pi^{*}(T)\right\|$ cohomologically. The cohomological classes of $\widetilde{K}_{n}^{ \pm}$are bounded, hence there exists a constant $c_{3}>0$ such that

$$
\left\|\widetilde{K}_{n}^{ \pm} \wedge \Pi^{*}(T)\right\| \leqslant c_{3}\|T\| .
$$

It follows that

$$
\left\|T_{n}^{ \pm}\right\|=\left\|\left(\pi_{1}\right)_{*} \pi_{*}\left(\widetilde{K}_{n}^{ \pm} \wedge \Pi^{*}(T)\right)\right\| \leqslant c\|T\|
$$

where $c>0$ is independent of $n$ and $T$.

The proof of Theorem 1.1 is completed by the following three steps.

Step 1. We show first that we can choose in Theorem 1.1 forms $T_{n}^{ \pm}$with $\mathrm{L}^{1}$ coefficients. Define $T_{n}^{ \pm}$as in (7) and (8). We use partitions of unity of $X$ and of $X \times X$ in order to reduce the problem to the case of $\mathbb{R}^{m}$. Following Lemmas 2.1 and 3.1, the forms $T_{n}^{ \pm}$have $\mathrm{L}^{1}$ coefficients. Lemma 3.2 implies that $T_{n}^{+}-T_{n}^{-} \rightarrow T$ and $\left\|T_{n}^{ \pm}\right\| \leqslant c\|T\|$. Of course, in general, $T_{n}^{+}-T_{n}^{-}$do not converge in $\mathrm{L}^{1}$ since the constants $A_{n}$ in Lemma 3.1 depend on $n$.

Step 2. We can now assume that $T$ is a form with $L^{1}$ coefficients. Define $T_{n}^{ \pm}$as in (7) and (8). Lemmas 2.1 and 3.1 imply that $T_{n}^{ \pm}$are forms with coefficients in $\mathrm{L}^{1+\delta}$. We also have $T_{n}^{+}-T_{n}^{-} \rightarrow T$ and $\left\|T_{n}^{ \pm}\right\| \leqslant c\|T\|$. Hence, we can assume that $T$ is a form with $\mathrm{L}^{1+\delta}$ coefficients. 
We repeat this process $N$ times with $N \geqslant \delta^{-1}$. Lemmas 2.1, 3.1 and 3.2 allow to reduce the problem to the case where $T$ is a form with $\mathrm{L}^{\infty}$ coefficients. If we repeat this process two more times, we can assume that $T$ is a $\mathcal{C}^{1}$ form.

Step 3. Now assume that $T$ is of class $\mathcal{C}^{1}$. We can also assume that $T$ is strictly positive. Let $\Omega$ be a smooth real closed $(p, p)$-form cohomologous to $T$. Using standard Hodge theory [6], there is a real $(p-1, p-1)$-form $u$ of class $\mathcal{C}^{2}$ such that $T=\Omega+\operatorname{dd}^{\mathrm{c}} u$. Let $\left(u_{n}\right)$ be a sequence of real smooth $(p-1, p-1)$-forms such that $u_{n} \rightarrow u$ in $\mathcal{C}^{2}$ norm. The current $T_{n}:=\Omega+\operatorname{dd}^{\mathrm{c}} u_{n}$ converges to $T$ in $\mathcal{C}^{0}$ norm. Moreover, $T_{n}$ is positive for $n$ big enough since $T$ is strictly positive. This completes the proof of Theorem 1.1.

\section{Pluriharmonic and DSH currents}

In this section, we extend Theorem 1.1 to positive pluriharmonic currents, i.e. positive $\mathrm{dd}^{\mathrm{c}}$-closed currents, and currents of class DSH. We have the following result which is new even for bidegree $(1,1)$ currents.

THEOREM 4.1. - Let $T$ be a positive $\mathrm{dd}^{\mathrm{c}}$-closed $(p, p)$-current on a compact Kähler manifold $(X, \omega)$. Then there exist smooth positive $\mathrm{dd}^{\mathrm{c}}$-closed forms $T_{n}^{ \pm}$such that $T_{n}^{+}-T_{n}^{-} \rightarrow T$. Moreover, $\left\|T_{n}^{ \pm}\right\| \leqslant c_{X}\|T\|$ where $c_{X}>0$ is a constant independent of $T$.

We deduce from this theorem the following corollary.

Corollary 4.2. - Let $X, X^{\prime}, \Pi, \pi$ and $\mathrm{C}$ be as in Corollary 1.3. If $T$ is as in Theorem 4.1, then the positive $\mathrm{dd}^{\mathrm{c}}$-closed current $\widetilde{\pi^{*}(T)}$ is well defined. Moreover the operator $T \mapsto \widetilde{\pi^{*}(T)}$ is l.s.c. and $\left\|\widetilde{\pi^{*}(T)}\right\| \leqslant c_{\Pi}\|T\|$ where $c_{\Pi}>0$ is a constant independent of $T$.

To prove the corollary, observe that by Theorem 4.1, the positive pluriharmonic current $\pi^{*}(T)$, which is well defined on $X^{\prime} \backslash \mathrm{C}$, has finite mass. Following Alessandrini and Bassanelli [1], $\widetilde{\pi^{*}(T)}$ satisfies $\mathrm{dd}^{\mathrm{c}} \widetilde{\pi^{*}(T)} \leqslant 0$. Then, Stokes Theorem implies that $\mathrm{dd}^{\mathrm{c}} \widetilde{\pi^{*}(T)}=0$.

Proof of Theorem 4.1. - We use the same idea as in Section 3. Clearly $T_{n}^{ \pm}$given by (7), (8) and (9) are pluriharmonic positive currents. We only need to check that $T_{n}^{+}-T_{n}^{-} \rightarrow T$. The rest of proof is the same as in Theorem 1.1.

Let $\varphi$ and $\varphi_{n}$ be quasi-p.s.h. functions as in Section 3. We want to prove the analog of (10):

$$
\left(\operatorname{dd}^{\mathrm{c}} \varphi_{n}+\Theta^{\prime}\right) \wedge \Pi^{*}(T) \rightarrow\left(\Pi_{\mid \widetilde{\Delta}}\right)^{*}(T) .
$$

The problem is local. Define $S:=\left(\Pi_{\mid \widetilde{\Delta}}\right)^{*}(T)$. We choose as in Lemmas 3.1 and 3.2 local holomorphic coordinates $\left(x_{1}, \ldots, x_{2 k}\right)$ of an open set $W \subset \widetilde{X \times X},\left|x_{i}\right|<1$, so that in $W$

- $\widetilde{\Delta}=\left\{x_{2 k}=0\right\}$; hence $\psi:=\varphi-\log \left|x_{2 k}\right|$ is smooth and $\mathrm{dd}^{\mathrm{c}} \psi=-\Theta^{\prime}$;

- $\Pi\left(x_{1}, \ldots, x_{2 k}\right)=\left(x_{1}, \ldots, x_{k}\right)$.

Define $\tau\left(x_{1}, \ldots, x_{2 k}\right):=\left(x_{1}, \ldots, x_{2 k-1}\right)$. Since $\Pi=\Pi_{\mid \widetilde{\Delta}} \circ \tau$, we have $\Pi^{*}(T)=\tau^{*}(S)$ in $W$.

Observe that $\left(\mathrm{dd}^{\mathrm{c}} \varphi_{n}+\Theta^{\prime}\right) \wedge \tau^{*}(S)$ is supported in $(\varphi<-n+2)$ and, by (6),

$$
\left(\operatorname{dd}^{\mathrm{c}} \varphi_{n}+\Theta^{\prime}\right) \wedge \tau^{*}(S) \geqslant\left(1-\chi_{n}^{\prime} \circ \varphi\right) \Theta^{\prime} \wedge \tau^{*}(S) .
$$

The definition of $\chi_{n}$ implies that the measures $\left(1-\chi_{n}^{\prime} \circ \varphi\right) \Theta^{\prime} \wedge \tau^{*}(S)$ tend to 0. Hence, every limit value of $\left(\mathrm{dd}^{\mathrm{c}} \varphi_{n}+\Theta^{\prime}\right) \wedge \tau^{*}(S)$ is a positive current $R$ supported in $\widetilde{\Delta}$. Following 
Bassanelli [2], it is a current on $\widetilde{\Delta}$ (this is true for every positive current $T$ supported in $\widetilde{\Delta}$ such that $\mathrm{dd}^{\mathrm{c}} R$ is of order 0 ). Hence, in order to prove (11) we only have to check that

$$
\int_{W} \Psi\left(x_{2 k}\right)\left(\operatorname{dd}^{\mathrm{c}} \varphi_{n}+\Theta^{\prime}\right) \wedge \tau^{*}(\Phi \wedge S) \rightarrow \int_{\widetilde{\Delta}} \Phi \wedge S
$$

for every test $(2 k-p-1,2 k-p-1)$-form $\Phi$ with compact support in $\widetilde{\Delta} \cap W$ and for every function $\Psi\left(x_{2 k}\right)$ supported in $\left\{\left|x_{2 k}\right|<1\right\}$, such that $\Psi(0)=1$. Observe that since $\tau^{*}(\Phi \wedge S)$ is proportional to $\mathrm{d} x_{1} \wedge \mathrm{d} \bar{x}_{1} \wedge \cdots \wedge \mathrm{d} x_{2 k-1} \wedge \mathrm{d} \bar{x}_{2 k-1}$ only the component of $\mathrm{dd}^{\mathrm{c}} \varphi_{n}+\Theta^{\prime}$ with respect to $\mathrm{d} x_{2 k} \wedge \mathrm{d} \bar{x}_{2 k}$ is relevant. When $\left(x_{1}, \ldots, x_{2 k-1}\right)$ is fixed, we have

$$
\int_{x_{2 k}} \Psi\left(\mathrm{dd}_{x_{2 k}}^{\mathrm{c}} \varphi_{n}+\Theta^{\prime}\right) \rightarrow 1
$$

since $\mathrm{dd}_{x_{2 k}}^{\mathrm{c}} \varphi_{n}+\Theta^{\prime}$ converges to the Dirac mass $\delta_{0}$ and $\Psi(0)=1$. The last integral is uniformly bounded with respect to $n$ and $x_{1}, \ldots, x_{2 k-1}$ because by (6) one can prove that the masses of the measures $\mathrm{dd}_{x_{2 k}}^{\mathrm{c}} \varphi_{n}+\Theta^{\prime}$ on a compact set of $\left\{\left|x_{2 k}\right|<1, x_{1}, \ldots, x_{2 k-1}\right.$ fixed $\}$ are uniformly bounded. This implies the result.

Remark 4.3. - Theorem 4.1 implies that on an arbitrary compact Kähler manifold $(X, \omega)$ positive pluriharmonic currents $T$ of bidegree $(1,1)$ have finite energy. We then have $T=$ $\Omega+\partial S+\overline{\partial S}+i \partial \bar{\partial} v$ with $\Omega$ smooth closed, $S, \partial S, \bar{\partial} S$ in $\mathrm{L}^{2}$ and $v$ in $\mathrm{L}^{1}$. The energy of $T$ is equal to $\int \bar{\partial} S \wedge \partial \bar{S} \wedge \omega^{k-2}$. The case of the projective space is treated in [14]. To extend the result to an arbitrary compact Kähler manifold, one has to use the approximation Theorem 4.1, to go from a priori estimates on smooth positive pluriharmonic forms to the estimates on positive pluriharmonic currents.

Let $\operatorname{DSH}^{p}(X)$ denote the space of $(p, p)$-currents $T=T_{1}-T_{2}$ where $T_{i}$ are negative currents, such that $\mathrm{dd}^{\mathrm{c}} T_{i}=\Omega_{i}^{+}-\Omega_{i}^{-}$with $\Omega_{i}^{ \pm}$positive closed. Observe that $\left\|\Omega_{i}^{+}\right\|=\left\|\Omega_{i}^{-}\right\|$. We define the $D S H$-norm of $T$ as

$$
\|T\|_{\mathrm{DSH}}:=\min \left\{\left\|T_{1}\right\|+\left\|T_{2}\right\|+\left\|\Omega_{1}^{+}\right\|+\left\|\Omega_{2}^{+}\right\|, T_{i}, \Omega_{i}^{ \pm} \text {as above }\right\} .
$$

We say that $T_{n} \rightarrow T$ in $\operatorname{DSH}^{p}(X)$ if $T_{n} \rightarrow T$ weakly and $\left(\left\|T_{n}\right\|_{\mathrm{DSH}}\right)$ is bounded.

The spaces $\operatorname{DSH}^{p}(X)$ are analogous to the space generated by quasi-p.s.h. functions. They are useful in order to study the regularity of Green currents in dynamics [11,12]. The proof of the following theorem, which gives the density of smooth forms in $\mathrm{DSH}^{p}(X)$, follows the lines of previous approximation results and is left to the reader. In this case, for the control of the mass of $T_{n}^{ \pm}$we need to estimate the mass of $\mathrm{dd}^{\mathrm{c}} \varphi_{n} \wedge \Pi^{*}\left(T_{i}\right)$. It is sufficient to estimate the mass of $\varphi_{n} \Pi^{*}\left(\mathrm{dd}^{\mathrm{c}} T_{i}\right)$ using the definition of $\varphi_{n}$.

THEOREM 4.4. - Let $T$ be a current in $\operatorname{DSH}^{p}(X)$. Then there exist smooth real $(p, p)$ forms $T_{n}$ such that $T_{n} \rightarrow T$. Moreover, $\left\|T_{n}\right\|_{\mathrm{DSH}} \leqslant c_{X}\|T\|_{\mathrm{DSH}}$ where $c_{X}>0$ is a constant independent of $T$.

Remark 4.5. - We have $\widetilde{K}_{n}^{+}-\widetilde{K}_{n}^{-}-\gamma \wedge[\widetilde{\Delta}]=\gamma \wedge \mathrm{dd}^{\mathrm{c}}\left(\varphi_{n}-\varphi\right)$ and $\varphi_{n}=\varphi$ out of the set $(\varphi<-n+2)$. Hence $\operatorname{supp}\left(\widetilde{K}_{n}^{+}-\widetilde{K}_{n}^{-}\right)$converge to $\widetilde{\Delta}, \operatorname{supp}\left(K_{n}^{+}-K_{n}^{-}\right)$converge to $\Delta$ and $\operatorname{supp}\left(T_{n}^{+}-T_{n}^{-}\right)$converge to $\operatorname{supp}(T)$.

We also have the following useful proposition. 
Proposition 4.6. - Let $T$ be a continuous form and $T_{n}^{ \pm}$be the forms defined in (7) and (8). Then $T_{n}:=T_{n}^{+}-T_{n}^{-}$converge uniformly to $T$. When $T$ is closed (resp. $\mathrm{dd}^{\mathrm{c}}$-closed) then $T_{n}^{ \pm}$ are closed (resp. $\mathrm{dd}^{\mathrm{c}}$-closed).

Proof. - We can approximate $T$ uniformly by smooth forms. We then assume that $T$ is smooth. The form $T_{n}-T$ is the push-forward of $\left(\widetilde{K}_{n}^{+}-\widetilde{K}_{n}^{-}-\gamma \wedge[\widetilde{\Delta}]\right) \wedge \Pi^{*}(T)$ by $\Pi^{\prime}:=\pi_{1} \circ \pi$ (see Lemma 3.1). The last current is equal to $\widetilde{T}_{n}:=\operatorname{dd}^{\mathrm{c}}\left(\varphi_{n}-\varphi\right) \wedge \gamma^{\prime}$ where $\gamma^{\prime}$ is a smooth form. Using a partition of unity, we reduce the problem to a local situation with the coordinates $x=\left(x^{\prime}, x^{\prime \prime}\right)=\left(x_{1}, \ldots, x_{k}, x_{k+1}, \ldots, x_{2 k}\right), \Pi^{\prime}(x)=x^{\prime}, \widetilde{\Delta}=\left(x_{2 k}=0\right)$ and $\gamma^{\prime}$ of compact support as in the proof of Theorem 4.1. We have to check that $\Pi_{*}^{\prime}\left(\widetilde{T}_{n}\right)\left(x^{\prime}\right)=\int_{x^{\prime \prime}} \widetilde{T}_{n}(x)$ converge uniformly to 0 .

Observe that the last integral is taken in the neighbourhood $(\varphi<-n+2)$ of $\left(x_{2 k}=0\right)$ and the form $\widetilde{T}_{n}-\mathrm{dd}_{x^{\prime \prime}}^{\mathrm{c}}\left(\varphi_{n}-\varphi\right) \wedge \gamma^{\prime}$ is of order $1 /\left|x_{2 k}\right|$ since in the difference we get at most one derivative with respect to $x_{2 k}$. Hence, it is sufficient to estimate

$$
\int_{x^{\prime \prime}} \operatorname{dd}_{x^{\prime \prime}}^{\mathrm{c}}\left(\varphi_{n}-\varphi\right) \wedge \gamma^{\prime}=\int_{x^{\prime \prime}}\left(\varphi_{n}-\varphi\right) \wedge \operatorname{dd}_{x^{\prime \prime}}^{\mathrm{c}} \gamma^{\prime}
$$

It is clear that these forms converge uniformly to 0 .

\section{Intersection of currents}

We want to consider a class of positive pluriharmonic currents which are of interest in some problems of complex analysis and dynamics. Some of their properties are given in $[21,1,2,13,9$, 14]. Given a compact Kähler manifold $(X, \omega)$ of dimension $k$, we want to define the intersection $S \wedge T$ of a positive closed $(1,1)$-current $S$ with a positive pluriharmonic current $T$ of bidegree $(p, p), 1 \leqslant p \leqslant k-1$. We have seen a special case of this situation in the last section.

We write $S=\alpha+\operatorname{dd}^{c} u$ with $\alpha$ smooth and $u$ a quasi-p.s.h. function. We say that $u$ is $a$ potential of $S$.

THEOREM 5.1. - Assume that $u$ is continuous. Then $S \wedge T$ is well defined and is a positive $\mathrm{dd}^{\mathrm{c}}$-closed current. Moreover $S \wedge T$ depends continuously on $S$ and $T$ in the following sense. Let $T_{n}$ be positive pluriharmonic currents converging weakly to $T$. If $S_{n}=\alpha+\mathrm{dd}^{\mathrm{c}} u_{n}$ with $u_{n}$ continuous converging uniformly to u then $S_{n} \wedge T_{n}$ converges weakly to $S \wedge T$. In particular, it holds when the $u_{n}$ are continuous and decrease to $u$.

We first prove the following proposition for smooth potentials. We will see later that it can be extended to continuous quasi-p.s.h. functions $v^{ \pm}$and that $\mathrm{d} v^{ \pm} \wedge R$ and $\mathrm{d}^{\mathrm{c}} v^{ \pm} \wedge R$ are well defined in this case.

Proposition 5.2. - Let $v^{ \pm}$and $v=v^{+}-v^{-}$be smooth real functions on $X$ such that $\operatorname{dd}^{\mathrm{c}} v^{ \pm}=\Theta^{ \pm}-\alpha$ where $\alpha$ is a smooth closed $(1,1)$-form and $\Theta^{ \pm}$are positive closed $(1,1)$-currents. Let $R$ be a positive current in $\operatorname{DSH}^{p}(X)$ with $\mathrm{dd}^{\mathrm{c}} R=\Omega^{+}-\Omega^{-}$where $\Omega^{ \pm}$ are positive closed currents. Then

$$
\int \mathrm{d} v \wedge \mathrm{d}^{\mathrm{c}} v \wedge R \wedge \omega^{k-p-1} \leqslant\|v\|_{\mathrm{L}^{\infty}}\left(2 \int \alpha \wedge R \wedge \omega^{k-p-1}+3\left(\left\|v^{+}\right\|_{\mathrm{L}^{\infty}}+\left\|v^{-}\right\|_{\mathrm{L}^{\infty}}\right)\left\|\Omega^{ \pm}\right\|\right) .
$$


In particular, if $R$ is positive pluriharmonic, we have

$$
\int \mathrm{d} v \wedge \mathrm{d}^{\mathrm{c}} v \wedge R \wedge \omega^{k-p-1} \leqslant 2\|v\|_{\mathrm{L}^{\infty}} \int[\alpha] \wedge[R] \wedge[\omega]^{k-p-1} .
$$

Proof. - Observe that $\|v\|_{L^{\infty}} \leqslant\left\|v^{+}\right\|_{L^{\infty}}+\left\|v^{-}\right\|_{L^{\infty}},\left\|\Theta^{+}\right\|=\left\|\Theta^{-}\right\|$and $\left\|\Omega^{+}\right\|=\left\|\Omega^{-}\right\|$. Hence

$$
\begin{aligned}
\int \mathrm{d} v & \mathrm{~d}^{\mathrm{c}} v \wedge R \wedge \omega^{k-p-1} \\
\leqslant & \frac{1}{2}\left|\int \mathrm{dd}^{\mathrm{c}} v^{2} \wedge R \wedge \omega^{k-p-1}\right|+\left|\int v \mathrm{dd}^{\mathrm{c}} v \wedge R \wedge \omega^{k-p-1}\right| \\
= & \frac{1}{2}\left|\int v^{2} \wedge \mathrm{dd}^{\mathrm{c}} R \wedge \omega^{k-p-1}\right|+\left|\int v\left(\Theta^{+}-\Theta^{-}\right) \wedge R \wedge \omega^{k-p-1}\right| \\
\leqslant & \frac{1}{2}\|v\|_{\mathrm{L}^{\infty}}^{2} \int\left(\Omega^{+}+\Omega^{-}\right) \wedge \omega^{k-p-1}+\|v\|_{\mathrm{L}^{\infty}} \int\left(\Theta^{+}+\Theta^{-}\right) \wedge R \wedge \omega^{k-p-1} \\
= & \|v\|_{\mathrm{L}^{\infty}}^{2}\left\|\Omega^{ \pm}\right\|+2\|v\|_{\mathrm{L}^{\infty}} \int \alpha \wedge R \wedge \omega^{k-p-1} \\
& +\|v\|_{\mathrm{L}^{\infty}} \int \mathrm{dd}^{\mathrm{c}}\left(v^{+}+v^{-}\right) \wedge R \wedge \omega^{k-p-1} \\
\leqslant & \|v\|_{\mathrm{L}^{\infty}}\left(\left\|v^{+}\right\|_{\mathrm{L}^{\infty}}+\left\|v^{-}\right\|_{\mathrm{L}^{\infty}}\right)\left\|\Omega^{ \pm}\right\|+2\|v\|_{\mathrm{L}^{\infty}} \int \alpha \wedge R \wedge \omega^{k-p-1} \\
& +\|v\|_{\mathrm{L}^{\infty}} \int\left(v^{+}+v^{-}\right) \wedge\left(\Omega^{+}-\Omega^{-}\right) \wedge \omega^{k-p-1} \\
\leqslant & \|v\|_{\mathrm{L}^{\infty}}\left(2 \int \alpha \wedge R \wedge \omega^{k-p-1}+3\left(\left\|v^{+}\right\|_{\mathrm{L}^{\infty}}+\left\|v^{-}\right\|_{\mathrm{L}^{\infty}}\right)\left\|\Omega^{ \pm}\right\|\right) .
\end{aligned}
$$

Proof of Theorem 5.1. - Observe that, when $u_{n}$ decreases to $u$, the Hartogs lemma implies that $u_{n}$ converges uniformly to $u$. By Demailly's regularization theorem [7], we can assume that $u_{n}$ is smooth and uniformly convergent to $u$. So, $S_{n} \wedge T_{n}$ is well defined. We will prove that $S_{n} \wedge T_{n}$ converges. This also implies that the limit depends only on $S$ and $T$.

We first consider the case where $T_{n}=T$. We then have

$$
S_{n} \wedge T=\alpha \wedge T+\mathrm{d}\left(\mathrm{d}^{\mathrm{c}} u_{n} \wedge T\right)-\mathrm{d}^{\mathrm{c}}\left(\mathrm{d} u_{n} \wedge T\right)-\mathrm{dd}^{\mathrm{c}}\left(u_{n} T\right) .
$$

Proposition 5.2 applied to $u_{n}-u_{m}$ and the Cauchy criterion imply that $\mathrm{d} u_{n}$ and $\mathrm{d}^{\mathrm{c}} u_{n}$ converge in $\mathrm{L}^{2}\left(T \wedge \omega^{k-p-1}\right)$. Hence $S_{n} \wedge T$ converges and $\mathrm{d} u \wedge T, \mathrm{~d}^{\mathrm{c}} u \wedge T$ are well defined.

To complete the proof we write

$$
S_{n} \wedge T_{n}-S \wedge T=\operatorname{dd}^{\mathrm{c}}\left(u_{n}-u\right) \wedge T_{n}+\mathrm{dd}^{\mathrm{c}} u \wedge\left(T_{n}-T\right)+\alpha \wedge\left(T_{n}-T\right) .
$$

The last term tends to zero. Proposition 5.2 implies that $\int \mathrm{d}\left(u_{n}-u\right) \wedge \mathrm{d}^{\mathrm{c}}\left(u_{n}-u\right) \wedge T_{n} \wedge \omega^{k-p-1}$ has zero limit. An identity as in (12) and Cauchy-Schwarz's inequality show that the first term tends to 0 . For the second term, we use again (12). We only need estimates of the following type. If $\gamma$ is a test 1 -form, $\Theta$ is a smooth positive $(k-p-1, k-p-1)$-form and $v$ is a smooth quasi-p.s.h. function with $\|u-v\|_{\mathrm{L}^{\infty}} \leqslant \epsilon$ and $\mathrm{dd}^{\mathrm{c}} v \geqslant-c \omega$, then 


$$
\begin{aligned}
\int \mathrm{d} u \wedge \gamma \wedge\left(T-T_{n}\right) \wedge \Theta= & \int \mathrm{d} v \wedge \gamma \wedge\left(T-T_{n}\right) \wedge \Theta \\
& +\int \mathrm{d}(u-v) \wedge \gamma \wedge\left(T-T_{n}\right) \wedge \Theta .
\end{aligned}
$$

The first integral tends to zero. Cauchy-Schwarz's inequality and Proposition 5.2 imply

$$
\begin{aligned}
\left|\int \mathrm{d}(u-v) \wedge \gamma \wedge T_{n} \wedge \Theta\right|^{2} & \leqslant \text { const } \int \mathrm{d}(u-v) \wedge \mathrm{d}^{\mathrm{c}}(u-v) \wedge T_{n} \wedge \Theta \\
& \leqslant \text { const }\|u-v\|_{\mathrm{L}^{\infty}}\left\|T_{n}\right\| \\
& \lesssim \epsilon
\end{aligned}
$$

and similarly for $T$.

In the same way, using the full strength of Proposition 5.2, one can prove the following theorem.

THEOREM 5.3. - Let $T$ be a current in $\operatorname{DSH}^{p}(X)$ and $S$ as in Theorem 5.1. Then $S \wedge T$ is well defined and belongs to $\mathrm{DSH}^{p+1}(X)$. Moreover $S \wedge T$ depends continuously on $S$ and $T$. The topology on the $T$ variable is the topology of $\operatorname{DSH}^{p}(X)$.

It is enough to assume $T$ positive and to modify (12) into

$$
S_{n} \wedge T=\alpha \wedge T+\mathrm{d}\left(\mathrm{d}^{\mathrm{c}} u_{n} \wedge T\right)-\mathrm{d}^{\mathrm{c}}\left(\mathrm{d} u_{n} \wedge T\right)-\operatorname{dd}^{\mathrm{c}}\left(u_{n} T\right)+u_{n} \mathrm{dd}^{\mathrm{c}} T .
$$

Remarks 5.4. - If $S_{i}$ are positive closed $(1,1)$-currents with continuous potentials, then $S_{1} \wedge \cdots \wedge S_{m} \wedge T$ is symmetric in $S_{i}$ since this is true when $S_{i}$ and $T$ are smooth. Let $u$ be a quasi-p.s.h. function on an open ball $\Omega \subset X$. By the maximum regularization procedure as in [6], if $u$ is continuous we can extend $u$ to a continuous quasi-p.s.h. function on $X$. Hence, $\operatorname{dd}^{\mathrm{c}} u \wedge T$ is well defined on $\Omega$.

When $T$ is only a (positive pluriharmonic) $(p, p)$-current on $\Omega$, we do not know how to define $\operatorname{dd}^{\mathrm{c}} u \wedge T$ without additional hypothesis on $u$. Assume that $T, \mathrm{~d} T$ and $\operatorname{dd}^{c} T$ are of order 0 and $u$ is locally integrable with respect to the coefficient measures of $T, \mathrm{~d} T$ and $\mathrm{dd}^{\mathrm{c}} T$. Then we can define

$$
\mathrm{dd}^{\mathrm{c}} u \wedge T:=\mathrm{dd}^{\mathrm{c}}(u T)+u \mathrm{dd}^{\mathrm{c}} T-\mathrm{d}\left(u \mathrm{~d}^{\mathrm{c}} T\right)+\mathrm{d}^{\mathrm{c}}(u \mathrm{~d} T) .
$$

If $u_{n}$ are p.s.h. and decrease to $u$ or if $u_{n}$ converge uniformly to $u$, we have

$$
\mathrm{dd}^{\mathrm{c}} u_{n} \wedge T \rightarrow \mathrm{dd}^{\mathrm{c}} u \wedge T
$$

When $T$ is positive, we also have an inequality of Chern-Levine-Nirenberg type (see [6, p. 126]). More precisely, if $K, L$ are compact sets in $\Omega$ with $L \Subset K$, then

$$
\left\|\mathrm{dd}^{\mathrm{c}} u \wedge T\right\|_{L} \leqslant c_{K, L}\left(\|u T\|_{K}+\|u \mathrm{~d} T\|_{K}+\left\|u \mathrm{dd}^{\mathrm{c}} T\right\|_{K}\right) .
$$

Note that positive harmonic currents associated to a lamination by Riemann surfaces satisfy the above hypothesis (see [3]).

If $T$ is of bidegree $(1,1)$ we can extend Theorem 5.1 to currents $S$ with bounded potential. 
Proposition 5.5. - Let $T$ be a positive pluriharmonic current of bidegree $(1,1)$ in $(X, \omega)$. If $u$ is a bounded quasi-p.s.h. function then $\mathrm{dd}^{\mathrm{c}} u \wedge T$ is well defined. If $\left(u_{n}\right)$ is a bounded sequence of quasi-p.s.h. functions converging pointwise to $u$ with $\mathrm{dd}^{\mathrm{c}} u_{n} \geqslant-c \omega$, then $\mathrm{dd}^{\mathrm{c}} u_{n} \wedge T \rightarrow \mathrm{dd}^{\mathrm{c}} u \wedge T$.

Proof. - We can assume that $u_{n}$ are smooth and positive. It is easy to check that $u_{n}^{2}$ are quasip.s.h. and converge to $u^{2}$. It follows that $\partial u_{n}$ (resp. $\bar{\partial} u_{n}$ ) converge to $\partial u$ (resp. $\bar{\partial} u$ ) weakly in $\mathrm{L}^{2}(X)$.

As in Theorem 5.1, we only need to show that $\partial u_{n} \wedge T$ (resp. $\bar{\partial} u_{n} \wedge T$ ) converges weakly. Recall that we can write $T=\Omega+\partial S+\overline{\partial S}+i \partial \bar{\partial} v$ with $\Omega$ smooth closed, $\partial S, \bar{\partial} S$ in $\mathrm{L}^{2}, v$ in $\mathrm{L}^{1}[14]$. If $\gamma$ is a test 1 -form, we have

$$
\begin{aligned}
\int \partial u_{n} \wedge T \wedge \gamma \wedge \omega^{k-1} & =-\int u_{n} \partial T \wedge \gamma \wedge \omega^{k-1}-\int u_{n} T \wedge \partial \gamma \wedge \omega^{k-1} \\
& =-\int u_{n} \partial \overline{\partial S} \wedge \gamma \wedge \omega^{k-1}-\int u_{n} T \wedge \partial \gamma \wedge \omega^{k-1}
\end{aligned}
$$

The second term tends to $\int u T \wedge \partial \gamma \wedge \omega^{k-1}$. The first term is equal to

$$
-\int \partial u_{n} \wedge \overline{\partial \bar{S}} \wedge \gamma \wedge \omega^{k-1}+\int u_{n} \overline{\partial S} \wedge \partial \gamma \wedge \omega^{k-1}
$$

which converges to

$$
-\int \partial u \wedge \overline{\partial S} \wedge \gamma \wedge \omega^{k-1}+\int u \overline{\partial S} \wedge \partial \gamma \wedge \omega^{k-1}
$$

since $u_{n} \rightarrow u$ and $\partial u_{n} \rightarrow \partial u$ weakly in $\mathrm{L}^{2}$.

The convergence of $\bar{\partial} u_{n} \wedge T$ is proved in the same way.

\section{REFERENCES}

[1] Alessandrini L., Bassanelli G., Plurisubharmonic currents and their extension across analytic subsets, Forum Math. 5 (6) (1993) 577-602.

[2] Bassanelli G., A cut-off theorem for plurisubharmonic currents, Forum Math. 6 (5) (1994) 567595.

[3] Berndtsson B., Sibony N., The $\bar{\partial}$ equation on a positive current, Invent. Math. 147 (2002) 371428.

[4] Blanchard A., Sur les variétés analytiques complexes, Ann. Sci. Éc. Norm. Sup. (3) 73 (1956) 157-202.

[5] Bowen R., Topological entropy for noncompact sets, Trans. Amer. Math. Soc. 184 (1973) 125-136.

[6] Demailly J.-P., Monge-Ampère operators, Lelong numbers and intersection theory, in: Ancona V., Silva A. (Eds.), Complex Analysis and Geometry, Plenum Press, 1993, pp. 115-193.

[7] Demailly J.-P., Pseudoconvex-concave duality and regularization of currents, in: Several Complex Variables (Berkeley, CA, 1995-1996), in: Math. Sci. Res. Inst. Publ., vol. 37, Cambridge Univ. Press, Cambridge, 1999, pp. 233-271.

[8] Dinh T.C., Distribution des préimages et des points périodiques d'une correspondance polynomiale, Bull. Soc. Math. France, in press.

[9] Dinh T.C., Lawrence M., Polynomial hull and positive currents, Ann. Fac. Sci. Toulouse XII (3) (2003) 317-334.

[10] Dinh T.C., Sibony N., Une borne supérieure pour l'entropie topologique d'une application rationnelle, Ann. of Math., in press. 
[11] Dinh T.C., Sibony N., Distribution des valeurs de transformations méromorphes et applications, preprint, 2003, math.DS/0306095.

[12] DinH T.C., Sibony N., Green currents for automorphisms of compact Kähler manifolds, JAMS, in press.

[13] Duval J., Sibony N., Polynomial convexity, rational convexity, and currents, Duke Math. J. 79 (2) (1995) 487-513.

[14] FoRnÆSS J.E., Sibony N., Harmonic currents with finite energy, preprint, 2004, math.CV/0402432.

[15] FRIEDLAND S., Entropy of polynomial and rational maps, Ann. of Math. 133 (1991) 359-368.

[16] Gromov M., On the entropy of holomorphic maps, in: Séminaire Bourbaki, vol. 1985/86, in: Astérique, vols. 145-146, Soc. Math. France, 1987, pp. 225-240.

[17] Gromov M., On the entropy of holomorphic maps, Enseign. Math. 49 (2003) 217-235, Manuscript (1977).

[18] LELONG P., Fonctions plurisousharmoniques et formes différentielles positives, Dunod, Paris, 1968.

[19] MÉo M., Image inverse d'un courant positif fermé par une application surjective, CRAS 322 (1996) $1141-1144$.

[20] Sibony N., Dynamique des applications rationnelles de $\mathbb{P}^{k}$, Panoramas et Synthèses 8 (1999) 97-185.

[21] Sibony N., Quelques problèmes de prolongement de courants en analyse complexe, Duke Math. J. 52 (1) (1985) 157-197.

[22] Skoda H., Prolongement des courants positifs, fermés de masse finie, Invent. Math. 66 (1982) 361376.

[23] Yomdin Y., Volume growth and entropy, Israel J. Math. 57 (1987) 285-300.

(Manuscrit reçu le 29 mars 2004 ; accepté, après révision, le 28 septembre 2004.)

\author{
Tien-Cuong DinH \\ Université Paris-Sud, \\ Mathématique - Bât. 425, \\ UMR 8628, \\ 91405 Orsay, France \\ E-mail: TienCuong.Dinh@math.u-psud.fr
}

\author{
Nessim Sibony \\ Université Paris-Sud, \\ Mathématique - Bât. 425, \\ UMR 8628 , \\ 91405 Orsay, France \\ E-mail: Nessim.Sibony@math.u-psud.fr
}

